\title{
Detection of the mandibular canal in orthopantomography using a Gabor-filtered anisotropic generalized Hough transform ${ }^{\text {th }}$
}

\author{
Darian Onchis-Moaca a,b,*, Simone Zappaláa, Smaranda Laura Goţiac , Pedro Real ${ }^{\text {, }}$ \\ Marius Pricop ${ }^{\mathrm{e}}$ \\ ${ }^{a}$ Faculty of Mathematics, University of Vienna, Oskar-Morgenstern-Platz 1, Vienna, Austria \\ ${ }^{\mathrm{b}}$ Department of Electrical Engineering and Informatics, Eftimie Murgu University of Resita, Traian Vuia Square 1-4, Resita, Romania \\ ${ }^{\mathrm{c}}$ Department of Physiology, Victor Babes University of Medicine and Pharmacy, Eftimie Murgu Street 2, Timisoara, Romania \\ ${ }^{\mathrm{d}}$ Department of Applied Mathematics I, University of Seville, Av. Reina Mercedes, Seville, Spain \\ e Department of Maxillofacial Surgery, Victor Babes University of Medicine and Pharmacy, Eftimie Murgu Street 2, Timisoara, Romania
}

\section{A R T I C L E I N F O}

Article history:

Available online 17 December 2015

\section{Keywords:}

Generalized Hough transform

Gabor transform

Mandibular canal

\begin{abstract}
A B S T R A C T
In this paper, we explore the possibility of applying the anisotropic generalized Hough transform (AGHT) enhanced with a Gabor based time-frequency filtering (GTF) for the determination of the mandibular canal in digital dental panoramic radiographs. The proposed method is based on template matching using the fact that the shape of the mandibular canal is usually the same, followed by a filtering of the accumulator space in the Gabor domain for a precise detection of the position. The proposed procedure consists of a detailed description of the shape of the canal in its canonical form and on preserving Gabor filtering information for sorting the hierarchy of location candidates after applying anisotropically the extraction algorithm. The experimental results show that the proposed procedure is robust to recognition under occlusion and under the presence of additional structures e.g. teeth, projection errors.
\end{abstract}

(c) 2015 Elsevier B.V. All rights reserved.

\section{Introduction}

The estimation of the mandibular canal is useful in detecting the nerve for inferior teeth called inferior dental nerve which is found inside it. While there are many research studies trying to visually identify the mandibular canal eg. $[1,7,9]$, the aim of this work is to propose an automatic method based on the anisotropic generalized Hough transform filtered with a Gabor-based transform for marking the mandibular canal in dental panoramic radiography. The clinical motivation relies in the ground fact that these types of images are relatively cheap and easy to acquire compared with other types of imagistic investigations e.g. computertomography and they are still widely spread within the community of oromaxillofacial practitioners.

On one side, the Hough transform is a popular technique to extract features from an image. The method was patented in 1962 [5] for the detection of lines in photographs. The functioning of the algorithm lies in a proper choice of the parameters space for the

\footnotetext{
This paper has been recommended for acceptance by Helena Molina-Abril.

* Corresponding author at: Faculty of Mathematics, University of Vienna, OskarMorgenstern-Platz 1, Vienna, Austria. Tel.: +43 14277506 97; fax: +43 14277506 90.

E-mail address: darian.onchis@univie.ac.at (D. Onchis-Moaca).
}

set of lines on the plane. Consider the implicit equation of a line as expressed in [6]

$f((\hat{m}, \hat{c}),(x, y))=y-\hat{m} x-\hat{c}=0$

where $\hat{m}$ and $\hat{c}$ are the slope and the intercept that characterize the line. Eq. (1) maps every pair of parameters $(\hat{m}, \hat{c})$ to a specific line, a shape.

By swapping the roles of the parameters and the points and fixing one point, the equation defines all the lines that pass through the fixed point.

So, if we consider some points in an image, they will be collinear if we can find a parameter $(\hat{m}, \hat{c})$ which satisfy $(1)$ for all the fixed points.

Following [2], consider now a generic curve in analytic form

$f(\boldsymbol{x}, \boldsymbol{a})=0$

where $\boldsymbol{x}$ is a point and $\boldsymbol{a}$ is a parameter vector. For every point we will find a hypersurface of parameters that satisfy (2). The curve will be detected in the intersection of these hypersurface. For example if we consider a circle

$\left(x-x_{0}\right)+\left(y-y_{0}\right)=r_{0}^{2}$,

due to the relation among $x_{0}, y_{0}$, and $r_{0}$, the number of free parameters is two. 


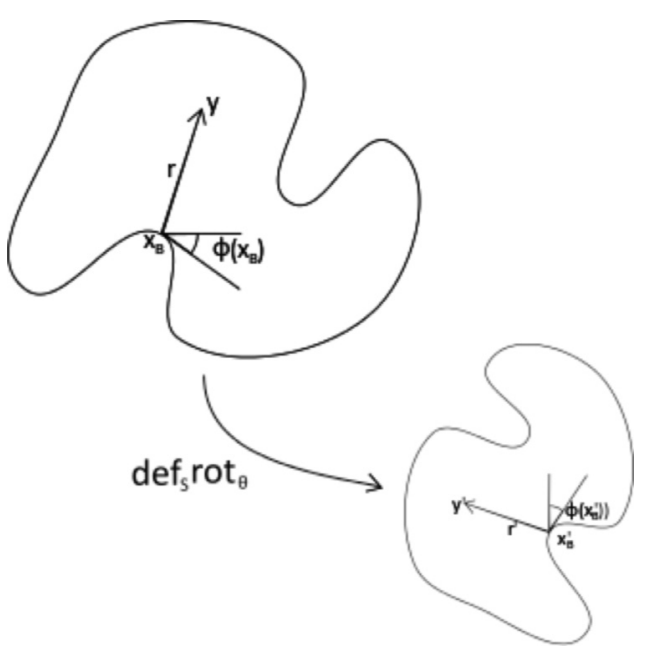

Fig. 1. Explanation for the notation used in the Hough transform method.

Every point $(x, y)$ will produce a cone in the parameter space $\left(x_{0}, y_{0}, r_{0}\right)$; if we consider three point on the plane, we can find the related circle as the intersection of these 3 cones.

Now we can add further information that can be extracted an image: the gradient and the tangent on the points of the edge. We can use the equation of the curve together with its derivative to find all the parameters which satisfy

$\frac{\partial f}{\partial \boldsymbol{x}}(\boldsymbol{x}, \boldsymbol{a})=0$

or similarly

$\frac{\partial y}{\partial x}=\tan \left(\phi(x)-\frac{\pi}{2}\right)$,

which is known since $\phi(x)$ is the gradient direction (see Fig. 1).

Going back to the example of the circle, fixing the gradient with the point means that the center must lie $r_{0}$ units along the direction of the gradient, so the number of free parameters reduces to one.

In order to develop a method for the recognition of a generic template in an image, [2] used the following parameters for a shape:

$\boldsymbol{a}=\{\boldsymbol{y}, \boldsymbol{S}, \theta\}$

where $\boldsymbol{y}=\left(x_{r}, y_{r}\right)$ is a reference point to represent the translations, $\boldsymbol{S}=\left(S_{x}, S_{y}\right)$ are scale values for the orthogonal shearing deformations, and $\theta$ is an angle that represents the rotations.

The reference point $\boldsymbol{y}$ is described in terms of a table, called the R-table (Table 1 ) of the template, of possible edge pixel orientations. The other parameters are described in terms of transformations of the aforementioned table.

The key for generalizing the Hough transform is to use the directional information. Given a template, i.e. a set of boundary points $\left\{\boldsymbol{x}_{B}\right\}$, a reference point $\boldsymbol{y}$ is chosen. After the discretization of the straight angle through a uniform partition $\{0, \Delta t$, $2 \Delta t, \ldots, N \Delta t\}$, for every boundary point the tangent direction $\phi\left(\boldsymbol{x}_{B}\right)$ is computed, then $\boldsymbol{r}=\boldsymbol{y}-\boldsymbol{x}_{B}$ is stored in the $n$th bin of the R-table if $\bmod \left(\phi\left(\boldsymbol{x}_{B}\right), \pi\right) \in[(n-1) \Delta t, n \Delta t)$. This choice of using the angle modulus $\pi$ will be made clear later.

Usually, to control the error in the computation of $\boldsymbol{r}$, the barycenter of the boundary point is chosen as reference point and $\boldsymbol{r}$ is stored in polar coordinates.

We obtain a structure in the form

Given this simple structure, if we define the rotation and shearing operators, i.e. $\operatorname{rot}_{\theta}$ and $\operatorname{def}_{\boldsymbol{S}}$, we can build the following procedure to detect the template in the set of edge points of any image

\begin{tabular}{|c|c|}
\hline Bin & Points \\
\hline 1 & $\left\{\boldsymbol{r} \mid \exists \overline{\boldsymbol{x}} \in\left\{\boldsymbol{x}_{B}\right\}\right.$ s.t. $\bmod (\phi(\overline{\boldsymbol{x}}), \pi) \in[0, \Delta t[\}$ \\
\hline 2 & $\left\{\boldsymbol{r} \mid \exists \overline{\boldsymbol{x}} \in\left\{\boldsymbol{x}_{B}\right\}\right.$ s.t. $\bmod (\phi(\overline{\boldsymbol{x}}), \pi) \in[\Delta t, 2 \Delta t[\}$ \\
\hline$\vdots$ & $\vdots$ \\
\hline$N$ & $\left\{\boldsymbol{r} \mid \exists \overline{\boldsymbol{x}} \in\left\{\boldsymbol{x}_{B}\right\}\right.$ s.t. $\left.\bmod (\phi(\overline{\boldsymbol{x}}), \pi) \in[(N-1) \Delta t, N \Delta t]\right\}$ \\
\hline
\end{tabular}

1. Fix a deformation $\boldsymbol{S}$ and a rotation $\theta$.

2. Compute the gradient of the edge points $\phi\left(\boldsymbol{x}_{e}\right)$.

3. Select the Bin in the R-table corresponding to $\operatorname{def}_{\boldsymbol{S}}\left(\operatorname{rot}_{\theta}\left(\phi\left(\boldsymbol{x}_{e}\right)\right)\right)$.

4. For every $\boldsymbol{r}$ in the selected Bin, report an occurrence of $\boldsymbol{y}^{\prime}=$ $\boldsymbol{x}_{e}+\operatorname{def}_{\boldsymbol{S}}\left(\operatorname{rot}_{\theta}(\boldsymbol{r})\right)$ as possible reference point.

In this way, we find the edge point that satisfy the non-analytic version of (2) and (3). The space of occurrences for the reference point is called Accumulator Space.

On the other side, the main tool for time-frequency analysis is the Short-Time Fourier Transform (STFT), defined for functions $f, g \in \boldsymbol{L}^{2}\left(\mathbb{R}^{d}\right)$ at $\lambda=(\alpha, \beta) \in \mathbb{R}^{2 d}$ by

$V_{g} f(\lambda)=V_{g} f(\alpha, \beta)=\left\langle f, M_{\beta} T_{\alpha} g\right\rangle=\langle f, \pi(\lambda) g\rangle$

where $T_{\alpha} f(t)=f(t-\alpha)$ is the translation (time shift) and $M_{\beta} f(t)=e^{2 \pi i \beta \cdot t} f(t)$ is the modulation (frequency shift). The operators $\pi(\lambda):=M_{\beta} T_{\alpha}$ are called time-frequency shifts and the set $\Lambda=\left\{\lambda ; \lambda=(\alpha, \beta) \in \mathbb{R}^{d} \times \widehat{\mathbb{R}}^{d}\right\}$ is a lattice.

Gabor analysis $[3,8,10]$ is a discrete version of the STFT and it is concerned with the representation of signals or images using a series consisting of time-frequency shifted copies of the given atom $g$. The Gabor system for analysis $\mathcal{G}(g, \Lambda)=\{\pi(\lambda) g ; \lambda \in \Lambda\}$ over the lattice $\Lambda$ consists of the translated and modulated versions of $g$, and it forms a frame for the space $L^{2}\left(\mathbb{R}^{d}\right)$, if and only if there exist $0<A \leq B<\infty$ (frame bounds) with

$A\|f\|^{2} \leq \sum_{\lambda \in \Lambda}|\langle f, \pi(\lambda) g\rangle|^{2} \leq B\|f\|^{2}$ for every $f \in L^{2}\left(\mathbb{R}^{d}\right)$,

With the interpretation proposed in Section 3.1, we will use the direct Gabor transform for ranking in the accumulator space of the AGHT.

\section{Recognition of the pattern: the case of mandibular canal}

In the previous section, we described a common way to detect a template using the AGTH. Problems arise when we have to deal with real images which could be corrupted by noise. That is the case of radiography where the structure of a bone is not well defined and where some part of the bone which would be detected can be disguised - even from the human eye - with unwanted details.

The position of the mandibular canal is described by medical indications as follows: the canal starts at the mandibular foramen in the middle part of the vertical ramus. It continues through the mandibular bone and ends in the menton foramen between apexes of the two inferior premolars.

This indication can become misleading if we analyze radiograph of a patient who has lost some teeth.

Any surgical intervention in the mandibular area must prevent any nerve injury. The injury of the nerve would result in prolonged local and lower lip anesthesia for a minimum period of six weeks. Estimating the position of the mandibular canal means knowing the position of the nerve and by this the surgeon can estimate the risks and to adapt the surgical procedure to the individual case.

In our test we used Fig. 2 to extract the template of the canal. The recognition performs well on the same panoramic radiograph 


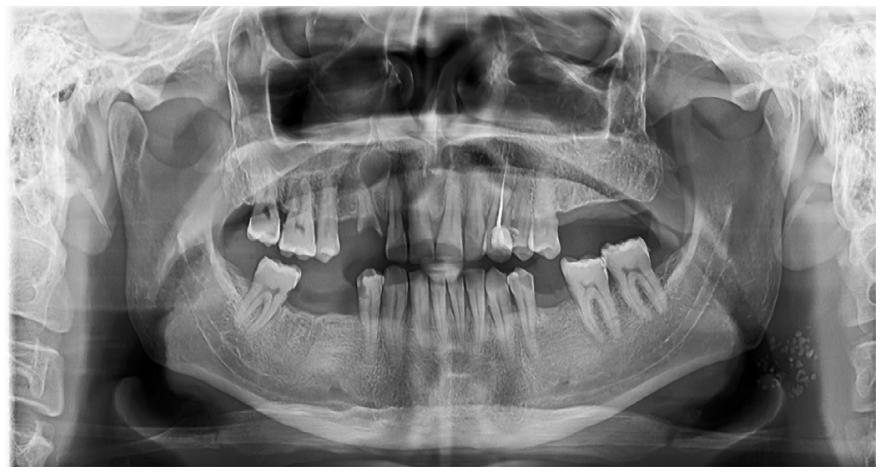

Fig. 2. Typical panoramic radiography.
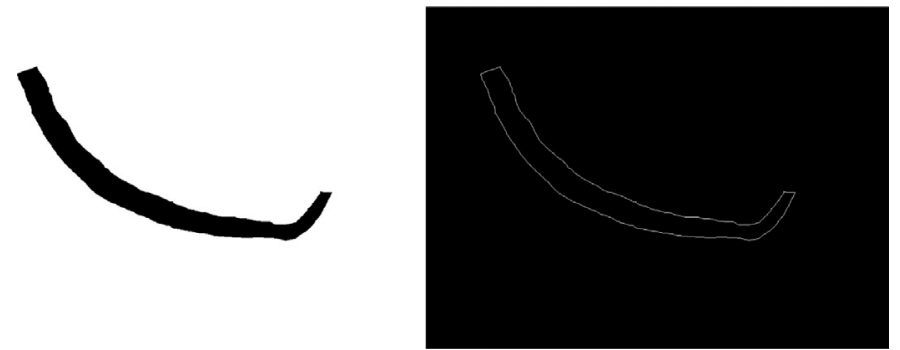

Fig. 3. Canal and its contour.

as it can be seen in Fig. 5, but the aforementioned image is in a critical situation: the patient has only one molar on the right part of the mandibular.

When the mandible is edentated, without any further restriction, the canal could not be recognized anymore because the AGHT algorithm matches the template in Fig. 3 with the top part of the alveolar process, the part of the bone where the teeth should be. It happens because the process is detected with a thicker edge than the canal, but has the same gradient direction, so in the recognition process it will have more importance. This undesired, unavoidable, matching has been soften by using the modulus- $\pi$ direction of the gradient: the canal, as modeled in Fig. 3, looks like the empty space in an edentated mouth; by using the modulus we remove every information about the inside-outside of the model. This seems the best choice for the case of the mandibular canal, a poorly defined region of the mandible enclosed by a slightly brighter contour.

This problem is related to how the AGHT is implemented: one of the weakness of this transform is that the accumulation space does not carry any information about the position in space of the template nor the mutual relation of different shape in the image. We identify three ways to overcome this problem:

- Manual solution: user should restrict the area to be investigated manually through anatomical information given beforehand.

- Semi-automatic solution: after a first, coarse and less accurate search for the mandible template through AGHT, the area to be investigated for the mandibular canal is automatically restricted.

- Automatic ranking of the accumulator space using the Gabor transform: as described in Section 3.1.

\section{Proposed method}

The canal template described in Section 2 has the following characteristics: it is a connected, compact and simply connected region of the plane. Therefore, the first step is to compute the edges. After the binarization of the template, we can run a contour-following algorithm to detect the boundary points. This way, one could reach the first purpose for the proposed pattern recognition: to obtain an easy manipulable set of data samples.

Nevertheless, the same strategy cannot be applied directly to a panoramic radiograph; also common edge detector filters such as Canny, Sobel, etc., fail. Radiographs are spurious images which contain a great amount of unwanted details. So, we concentrated our work on the choice of the good parameters for the detection of the edges of the teeth (for a good survey see [4]). This means the proper choice of the variance in the Gaussian filter and the threshold parameter.

The processes of low pass filtering and thresholding, cancel the mandibular canal from the image; so we have to create an ad hoc method for detecting the edges. It can be seen from Fig. 2 that mandibular canals are drawn by two bright gray curved lines in a darker gray background; the good point is that the orientation of the canal is steady. Therefore, the natural idea is to use a high pass anisotropic filter mask adjusted on the shape of the mandibular canal.

Pursuing this idea, after an histogram equalization, we used the following mask as anisotropic high pass filter (AHPF) to detect the right mandibular canal

$$
\begin{aligned}
A_{0} & =\left(\begin{array}{ccccc}
-\frac{5}{4} & -\frac{5}{4} & -\frac{5}{4} & -\frac{5}{4} & -\frac{5}{4} \\
\frac{1}{4} & \frac{1}{4} & \frac{1}{4} & \frac{1}{4} & \frac{1}{4} \\
2 & 2 & 2 & 2 & 2 \\
\frac{1}{4} & \frac{1}{4} & \frac{1}{4} & \frac{1}{4} & \frac{1}{4} \\
-\frac{5}{4} & -\frac{5}{4} & -\frac{5}{4} & -\frac{5}{4} & -\frac{5}{4}
\end{array}\right) \\
A_{1} & =\left(\begin{array}{ccccc}
2 & \frac{1}{4} & -1 & -1 & -1 \\
\frac{1}{4} & 2 & \frac{1}{4} & -1 & -1 \\
-1 & \frac{1}{4} & 2 & \frac{1}{4} & -1 \\
-1 & -1 & \frac{1}{4} & 2 & \frac{1}{4} \\
-1 & -1 & -1 & \frac{1}{4} & 2
\end{array}\right) \\
A_{2} & =\left(\begin{array}{ccccc}
{[1} & 2 & -\frac{1}{4} & -1 & -2 \\
0 & 2 & 1 & -\frac{1}{2} & -2 \\
-1 & -\frac{1}{4} & 2 & -\frac{1}{4} & -1 \\
-2 & -\frac{1}{2} & 1 & 2 & 0 \\
-2 & -1 & -\frac{1}{4} & 2 & 1
\end{array}\right)
\end{aligned}
$$

$A_{3}=A_{2}^{T}$

and their flipped left-to-right version for the left mandibular canal.

To calculate the gradient needed in the implementation of the AGHT we used a Sobel mask for both the boundary and the edge points to have consistency in the calculation.

After the calculation of the barycenter of the template, for the construction of the R-table we chose to store $\boldsymbol{r}=\boldsymbol{y}-\boldsymbol{x}_{B}$ in Cartesian coordinates to follow the natural discretization introduced by an image. This also helps us to understand the worst case scenarios: after the recognition process the accumulator space will be a matrix with the same dimension of the image so we could print it on screen in grayscale to understand how the error spread and which other shape can be disguised as a mandibular canal (see Fig. 4).

There are many ways to increment the accumulator space. The general command is

$A(\boldsymbol{a}):=A(\boldsymbol{a})+g(\boldsymbol{x})$

for all the different edge point $\boldsymbol{x}$. 


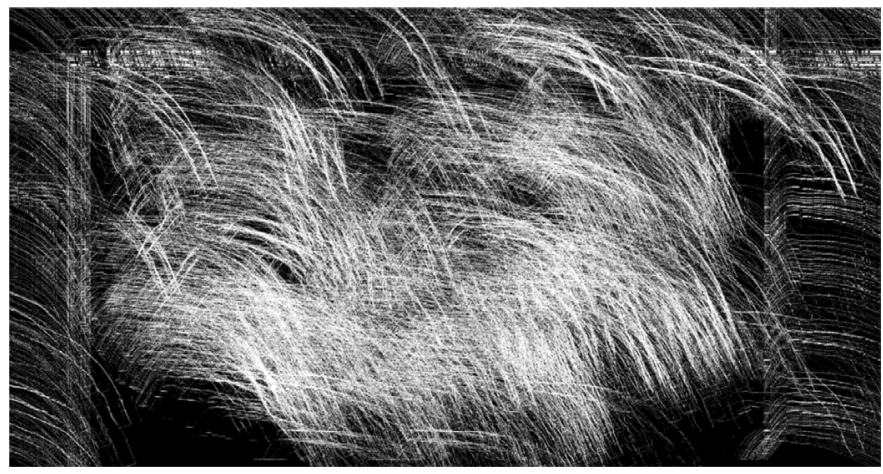

Fig. 4. Accumulator space for canal recognition.

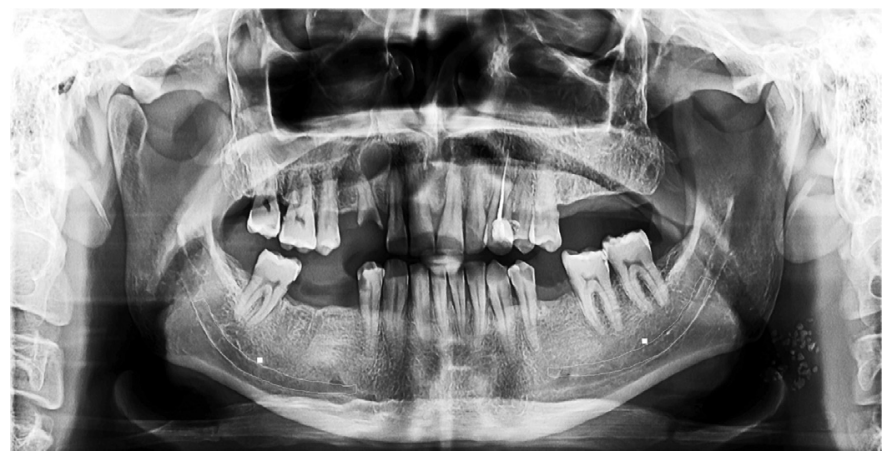

Fig. 5. Perfect matching of the template on the original image. Optimal matching of the flipped-left template.

By looking at the regularity of the mandibular canal we chose

$A(\boldsymbol{a})= \begin{cases}A(\boldsymbol{a})+5 & \text { if } \phi(\boldsymbol{x}) \in] \frac{1}{12} \pi, \frac{5}{12} \pi[ \\ A(\boldsymbol{a})+1 & \text { elsewhere }\end{cases}$

for the right canal and an analogous function for the left one.

The last remark should be about the parameters expressed in (4). We have not used the rotational parameter $\theta$ inasmuch as every panoramic radiography is taken with the patient's head fixed. The important parameter is $\boldsymbol{S}=\left(S_{x}, S_{y}\right)$ which helps us to reconstruct the anatomical difference among human beings. In this way, it is possible to find the best deformed version of the template in Fig. 3 that matches the canal in the panoramic radiograph under analysis.

\subsection{Gabor ranking of the accumulator space}

In order to propose a ranking for the best match in the accumulator space, we will use the Gabor transform with the following interpretation: instead of seeing $\omega_{1}$ and $\omega_{2}$ as the components of a 2D frequency, one might prefer to see $\omega$ as the parameter for the pattern density and define an orientation similar to the argument of complex numbers.

In order to detect the position of the best match in the accumulator space of the GHT for the reconstruction of the mandibular canal, we will perfom a Gabor transform to obtain local characteristics of the waves' intensities.

The localization of the image is done by computing the point wise multiplication $\mathrm{Im} \cdot \mathrm{T}_{\mathbf{l}}$ Atom for a set of different translation parameters $\left(T_{\mathbf{l}} \text { Atom }\right)_{\mathbf{k}}=$ Atom $_{k-l}$. The last step is the computation of the FFT ((Fast Fourier Transform)) for all these localized blocks. The drawback of this representation is that it considers a continuous domain. When discretized directly it results a highly redundant representation. Therefore, the proper sampling of position space and frequency space with step widths $\alpha=\left(\alpha_{1}, \alpha_{2}\right)$ and

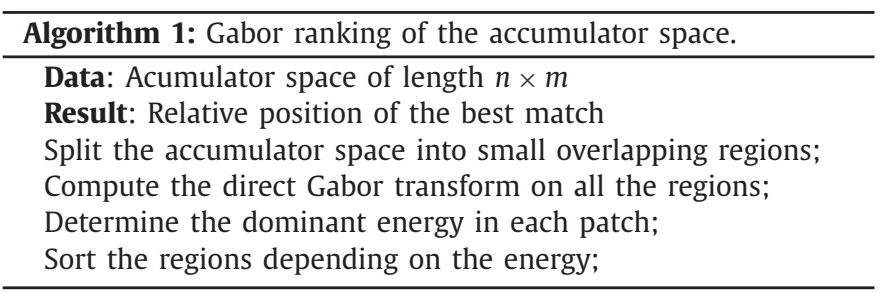

$\beta=\left(\beta_{1}, \beta_{2}\right)$, chosen such that we obtain a Gabor frame [10] is necessary. The result is a set of Gabor coefficients which represent local waves contained in the image.

As a localized and sampled 2D short-time Fourier transform, the Gabor representation, using discrete frames will give us, at each point of the lattice, a two-dimensional spectral representation of the image ordered either with modulation or with translation priority. Using this localized time-frequency representation, the harmonic filtering in phase-space will identify the pattern in the phase arguments.

In order to detect the best match, we first chop the accumulator space into small overlapping hexagonal regions in order to sample using the quincunx lattice. These regions are determined by their geometric coordinates in the accumulator space. Then we compute the Gabor representation on these patches for the first ranking. In the last step, we check if any energy variation happen in similar patches and detect the position of the best match by narrowing the analyzing window. This task is computationally expensive and high performance machines are recommended.

The procedure is presented in detail in Algorithm 1 .

\subsection{The algorithm}

We sum up in Algorithm 2 the complete procedure for the recognition of the mandibular canal, incorporating the AGTH detection and the GTF ranking.

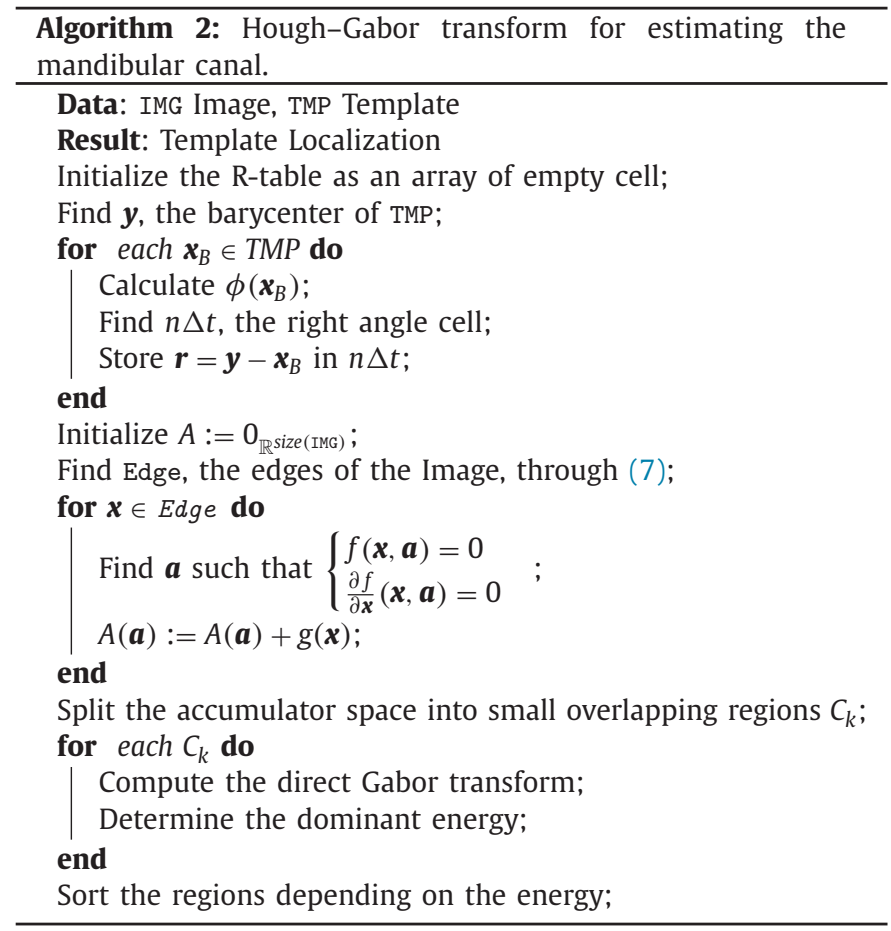




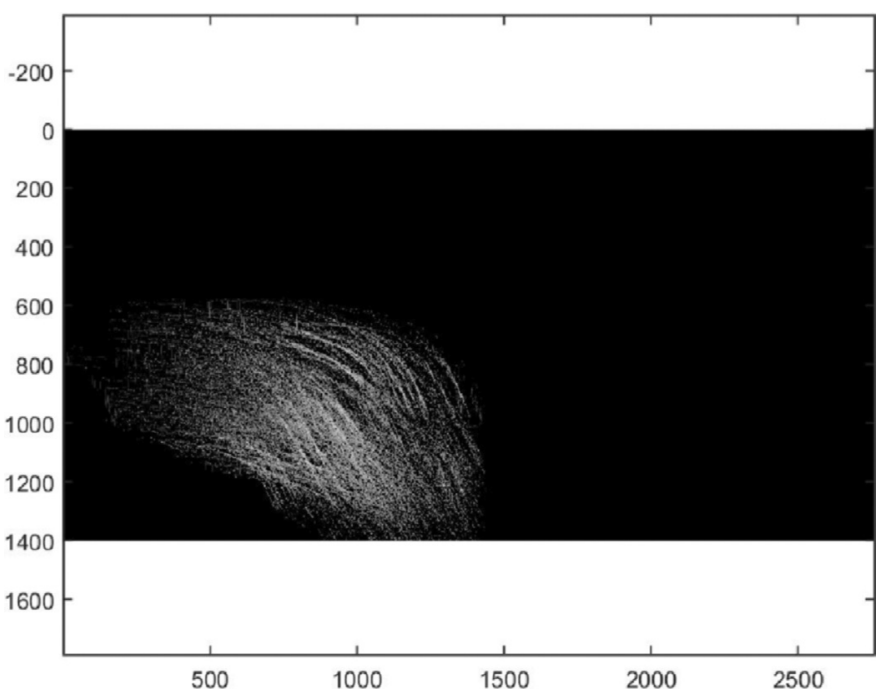

Fig. 6. Accumulator space for mandibular canal. Restriction to bottom-left part of picture (7).

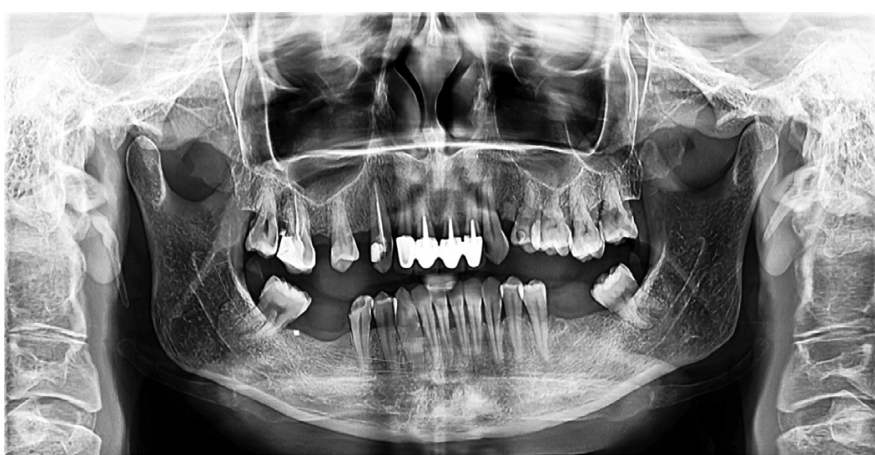

Fig. 7. Semi-automatic solution without area restriction.

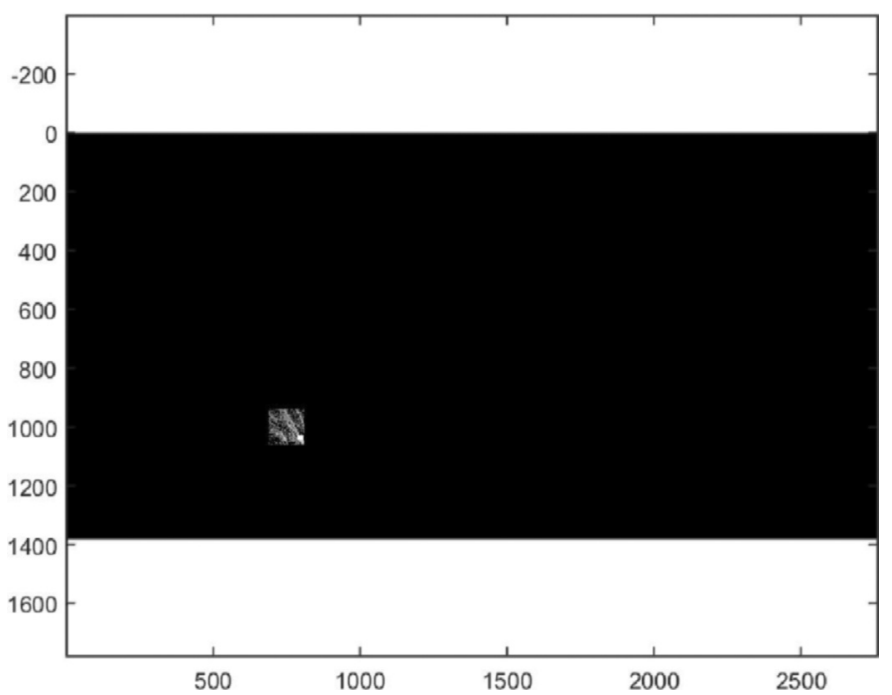

Fig. 8. Accumulator space for mandibular canal. Semi-automatic restriction.

\section{Results}

In this section, we present the experimental results of the proposed algorithm. The first test is to search for the template extracted from Fig. 2 in the same image. The overlapping is perfect even without any technique of region restriction. The same argument can be brought forward for the flipped template. Since there

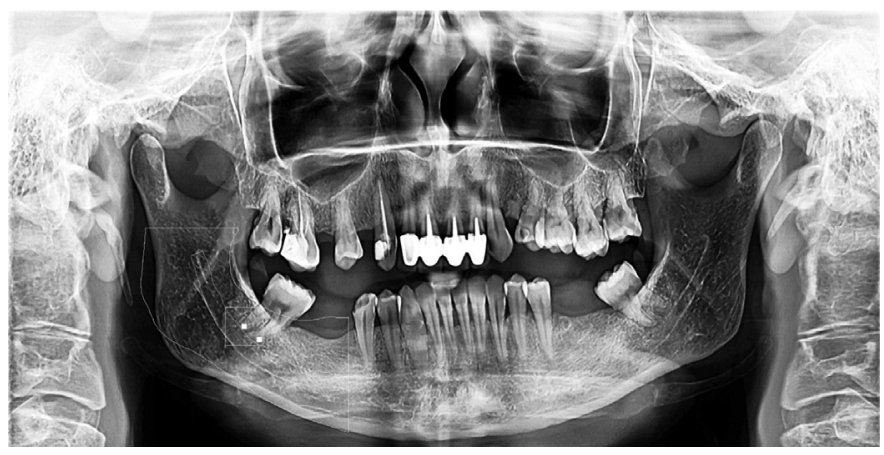

Fig. 9. Semi-automatic solution with area restriction.

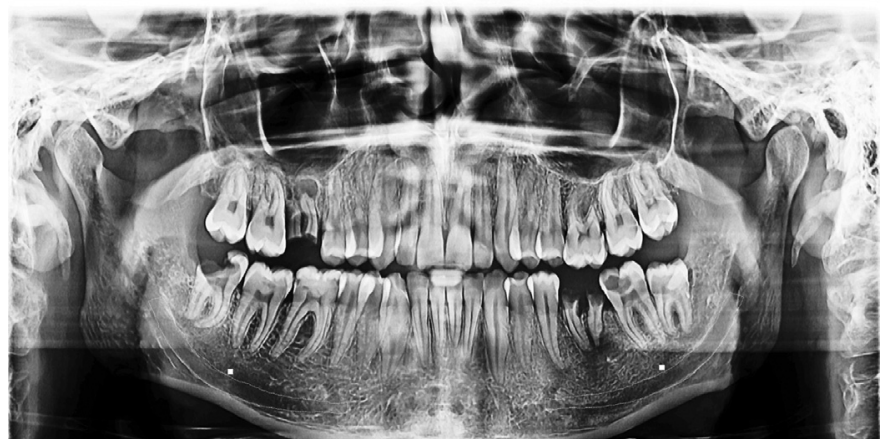

Fig. 10. Recognition of the template in Fig. 3 with scale parameters $\left(S_{x}, S_{y}\right)=$ $(1.1,1.15)$.

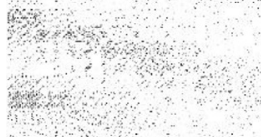

Fig. 11. GTF ranking (automatic area delimitation on the right side of the image).

is no significant anatomical difference between the left and the right side of the same patient, the overlapping is almost perfect.

As presented in Section 2, one of the biggest problems we encountered is the mismatch of the canal template with an edentated mandible where we had restricted the edge processing to the leftbottom part of the radiography (see Fig. 6).

We already identified in Section 2 three ways to overcome the problem of mismatching namely manual, semi-automatic, and automatic solution. A comparison could be summarized as follows: the first approach is as expected the worst solution because it is necessary to adjust the region of interest image by image (see Fig. 8).

The second approach is the semi-automatic solution, named in this way because it searches for the whole mandible to find the area of interest, then the AGHT recognition process for the canal is performed.

This process is possible because the canal lies in the center of the mandible, so their reference points (their barycenters) are really close. After the detection of reference points for the mandible $\boldsymbol{y}_{M}=\left(x_{M}, y_{M}\right)$, we force the reference points of the canal to be in the square window $\left[x_{M}-50, x_{M}+50\right] \times\left[x_{M}-50, x_{M}+50\right]$, with underlining unit of measure the pixel (Figs. 7 and 9).

It is easy observe in the Fig. 10 that if the patient has all his teeth, the area-restriction process is not required for the canal recognition. 
The automatic solution is to run completely the Algorithm 2 including the AGTH and the GTF ranking. In Fig. 11, one can see after applying the GTF ranking, the restriction area is automatically detected such that the AGTH reconstruction could be performed for the best match.

\section{Conclusions}

In this work, we used the AGHT for the detection of the mandibular canal in a panoramic radiograph, followed by a GTF filtering of the accumulator space. We have also indicated the problems we encountered in this particular case of pattern recognition:

- choice of parameter space

- edge detection

- zonal restriction

The first issue was solved through the choice of Cartesian coordinates for a better understanding of the local maximas. The second issue was resolved through a proper choice of the anisotropic high pass filter.

We had to deal with different shapes which can be mistaken with each other, such as the top and the bottom of the mandible.

The initial strategy was to restrict the area to be analyzed using medical information. This is not an optimal strategy for the application of the AGHT recognition process in general situations.

Therefore, we have used an automatic selection based on GTF ranking of the accumulator space for finding the best template match.

\section{Acknowledgments}

The first two co-authors gratefully acknowledge the support of the Austrian Science Fund (FWF): project number P27516.

\section{References}

[1] M.A. Atieh, Diagnostic accuracy of panoramic radiography in determining relationship between inferior alveolar nerve and mandibular third molar, J. Ora Maxillof. Surg. 68 (2010) 74-82.

[2] D.H. Ballard, Generalizing the Hough transform to detect arbitrary shapes, Pattern Recognit. 13 (1981) 111-122.

[3] H.G. Feichtinger, T. Strohmer, Gabor Analysis and Algorithms: Theory and Applications, Birkhäuser, 1998.

[4] L. Gráfová, M. Kašparová, S. Kakawand, A. Procházka, T. Dostálová, Study of edge detection task in dental panoramic radiographs, Dentomaxillofacial Radiol. 42 (2013) 20120391.

[5] V.C. Hough Paul, Method and means for recognizing complex patterns, 1962, US Patent 3069654. http://www.google.com/patents/US3069654.

[6] J. Illingworth, J. Kittler, A survey of the Hough transform, Comput. Vis. Graph. Image Process. 44 (1988) 87-116.

[7] A. Jhamb, R.S. Dolas, P.K. Pandilwar, S. Mohanty, Comparative efficacy of spiral computed tomography and orthopantomography in preoperative detection of relation of inferior alveolar neurovascular bundle to the impacted mandibular third molar, J. Oral Maxillofac. Surg. 67 (2009) 58-66.

[8] G. Karlheinz, Foundations of Time-Frequency Analysis, Birkhäuser, 2001.

[9] A. Mehra, K.M. Pai, Evaluation of dimensional accuracy of panoramic crosssectional tomography, its ability to identify the inferior alveolar canal, and its impact on estimation of appropriate implant dimensions in the mandibular posterior region, Clin. Implant Dent. Relat. Res. 14 (2012) 100-111.

[10] D.M. Onchis, P. Real, G.R. Gillich, Gabor frames and topology-based strategies for astronomical images, in: Proceedings of the CTIC 2010 Computational Topology in Image Context, Chipiona, Spain, 2010, pp. 159-166. 